\title{
Lattices Associated with a Finite Vector Space
}

\author{
Mengtian Yue \\ Office of Scientific Research, Langfang Teachers' College, Langfang, China \\ Email:ymtxyz@126.com
}

Received 23 December 2013; revised 23 January 2014; accepted 30 January 2014

Copyright @ 2014 by author and Scientific Research Publishing Inc.

This work is licensed under the Creative Commons Attribution International License (CC BY). http://creativecommons.org/licenses/by/4.0/

(c) (i) Open Access

\section{Abstract}

Let $F_{q}^{n}$ be a $n$-dimensional row vector space over a finite field $F_{q^{*}}$. For $1 \leq d \leq n-1$, let $W_{0}$ be a $d$ dimensional subspace of $F_{q}^{n}$. $L(n, d)$ denotes the set of all the spaces which are the subspaces of $F_{q}^{n}$ and not the subspaces of $W_{0}$ except $\{0\}$. We define the partial order on $L(n, d)$ by ordinary inclusion (resp. reverse inclusion), and then $L(n, d)$ is a poset, denoted by $L_{o}(n, d)$ (resp. $L_{R}(n, d)$ ). In this paper we show that both $L_{O}(n, d)$ and $L_{R}(n, d)$ are finite atomic lattices. Further, we discuss the geometricity of $L_{O}(n, d)$ and $L_{R}(n, d)$, and obtain their characteristic polynomials.

\section{Keywords}

Vector Space; Geometric Lattice; Characteristic Polynomial

\section{Introduction}

Let $P$ be a poset. For $a, b \in P$, we say $a$ covers $b$, denoted by $b<\cdot a$; if $b<a$ and there doesn't exist $c \in P$ such that $b<c<a$. If $P$ has the minimum (resp. maximum) element, then we denote it by 0 (resp. 1 ) and say that $P$ is a poset with 0 (resp. 1). Let $P$ be a finite poset with 0 . By a rank function on $P$, we mean a function $r$ from $P$ to the set of all the integers such that $r(0)=0$ and $r(a)=r(b)+1$ whenever $b<\cdot a$. Observe the rank function is unique if it exists. $P$ is said to be ranked whenever $P$ has a rank function.

Let $P$ be a finite ranked poset with 0 and 1 . The polynomial $\chi(P, x)=\sum_{a \in P} \mu(0, a) x^{r(1)-r(a)}$ is called the characteristic polynomial of $P$, where $\mu$ is the Möbius function on $P$ and $r$ is the rank function of $P$. A poset $P$ is said to be a lattice if both $a \vee b:=\sup \{a, b\}$ and $a \wedge b:=\inf \{a, b\}$ exist for any two elements $a, b \in P$. $a \vee b$ and $a \wedge b$ are called the join and meet of $a$ and $b$, respectively. Let $P$ be a finite lattice with 0 . By an 
atom in $P$, we mean an element in $P$ covering 0 . We say $P$ is atomic if any element in $P \backslash\{0\}$ is the join of atoms. A finite atomic lattice $P$ is said to be a geometric lattice if $P$ admits a rank function $r$ satisfying $r(a \wedge b)+r(a \vee b) \leq r(a)+r(b), \forall a, b \in P$. Notations and terminologies about posets and lattices will be adopted from books [1] [2].

The special lattices of rough algebras were discussed in [3]. The lattices generated by orbits of subspaces under finite (singular) classical groups were discussed in [4] [5]. Wang et al. [6]-[8] constructed some sublattices of the lattices in [4]. The subspaces of a $d$-bounded distance-regular have similar properties to those of a vector space. Gao et al. [9]-[11] constructed some lattices and posets by subspaces in a $d$-bounded distance-regular graph. In this paper, we continue this research, and construct some new sublattices of the lattices in [4], discussing their geometricity and computing their characteristic polynomials.

Let $F_{q}$ be a finite field with $q$ elements, where $q$ is a prime power. For a positive integer $n$, let $F_{q}^{n}$ be the $n$-dimensional row vector space over $F_{q}$. Let $1 \leq d \leq n-1$. For a fixed $d$-dimensional subspace $W_{0}$ of $F_{q}^{n}$, let $L(n, d)=\left\{P \mid P\right.$ is a subspace of $F_{q}^{n}$ and is not of $\left.W_{0}\right\} \cup\{\{0\}\}$.

If we define the partial order on $L(n, d)$ by ordinary inclusion (resp. reverse inclusion), then $L(n, d)$ is a poset, denoted by $L_{O}(n, d)$ (resp. $L_{R}(n, d)$ ). In the present paper we show that both $L_{O}(n, d)$ and $L_{R}(n, d)$ are finite atomic lattices, discuss their geometricity and compute their characteristic polynomials.

\section{The Lattice $L_{O}(n, d)$}

In this section we prove that the lattice $L_{O}(n, d)$ is a finite geometric lattice, and compute its characteristic polynomial. We begin with a useful proposition.

Proposition 2.1. ([12], Lemma 9.3.2 and [13], Corollaries 1.8 and 1.9). For $0 \leq k \leq m \leq n$, the following hold:

1) The number of $k$-dimensional subspaces contained in a given $m$-dimensional subspace of $F_{q}^{n}$ is

$$
\left[\begin{array}{l}
m \\
k
\end{array}\right]_{q}=\prod_{i=m-k-1}^{m}\left(q^{i}-1\right) / \prod_{i=1}^{k}\left(q^{i}-1\right) .
$$

2) The number of $m$-dimensional subspaces containing a given $k$-dimensional subspace of $F_{q}^{n}$ is

$$
\left[\begin{array}{c}
n-k \\
m-k
\end{array}\right]_{q}
$$

3) Let $P$ be a fixed $m$-dimensional subspaces of $F_{q}^{n}$. Then the number of $k$-dimensional subspaces $Q$ of $F_{q}^{n}$ satisfying $\operatorname{dim}(P \cap Q)=t$ is

$$
q^{(m-t)(k-t)}\left[\begin{array}{c}
n-m \\
k-t
\end{array}\right]_{q}\left[\begin{array}{c}
m \\
t
\end{array}\right]_{q} .
$$

Theorem 2.2. $L_{O}(n, d)$ is a geometric lattice.

Proof. For any two elements $P, Q \in L_{O}(n, d)$,

$$
P \vee Q=P+Q, P \wedge Q= \begin{cases}P \cap Q & \text { if } P \cap Q \not \subset W ; \\ \{0\} & \text { otherwise. }\end{cases}
$$

Therefore $L_{O}(n, d)$ is a finite lattice. Note that $\{0\}$ is the unique minimum element. Let $P(n, d ; j)$ be the set of all the $j$-dimensional subspaces of $L_{O}(n, d)$, where $1 \leq j \leq n$. Then $P(n, d ; 1)$ is the set of all the atoms in $L_{O}(n, d)$. In order to prove $L_{O}(n, d)$ is atomic, it suffices to show that every element of $P(n, d ; j)(1 \leq j \leq n)$ is a join of some atoms. The result is trivial for $j=1$. Suppose that the result is true for $j=l>1$. Let $U \in P(n, d ; l+1)$. By Proposition 2.1 and $\operatorname{dim}\left(W_{0} \cap U\right) \leq l$, the number of $l$-dimensional subspaces of $L_{O}(n, d)$ contained in $U$ at least is

$$
\left[\begin{array}{c}
l+1 \\
l
\end{array}\right]_{q}-1=\frac{q\left(q^{l}-1\right)}{q-1} \geq 2 .
$$

Therefore there exist two different $l$-dimensional subspaces $U^{\prime}, U^{\prime \prime} \subseteq U$ of $L_{O}(n, d)$ such that $U=U^{\prime} \vee U^{\prime \prime}$. 
By induction $U$ is a join of some atoms. Hence $L_{O}(n, d)$ is a finite atomic lattice. For any $U \in L_{O}(n, d)$, define $r_{O}(U)=\operatorname{dim} U$. It is routine to check that $r_{O}$ is the rank function on $L_{O}(n, d)$. For any $U, V \in L_{O}(n, d)$, we have

$$
\begin{aligned}
r_{O}(U \vee V)+r_{O}(U \wedge V) & =\operatorname{dim}(U+V)+\operatorname{dim}(U \wedge V) \\
& \leq \operatorname{dim}(U+V)+\operatorname{dim}(U \cap V) \\
& =\operatorname{dim} U+\operatorname{dim} V=r_{O}(U)+r_{O}(V) .
\end{aligned}
$$

Hence $L_{O}(n, d)$ is a geometric lattice.

Lemma 2.3. For any $P, Q \in L_{O}(n, d)$, suppose that $\operatorname{dim} P=t, \operatorname{dim} Q=t+s$ and $\operatorname{dim}\left(W_{0} \cap Q\right)=m$. Then the Möbius function of $L_{O}(n, d)$ is

$$
\mu(P, Q)= \begin{cases}(-1)^{s} q^{\left(\begin{array}{c}
s \\
2
\end{array}\right)} & \text { if }\{0\} \neq P \leq Q \text { or } P=Q=\{0\} ; \\
\sum_{l=1}^{s}(-1)^{s-l+1}\left(\left[\begin{array}{l}
s \\
l
\end{array}\right]_{q}-\left[\begin{array}{c}
m \\
l
\end{array}\right]_{q}\right) q^{\left(\begin{array}{c}
s-l \\
2
\end{array}\right)} & \text { if }\{0\}=P<Q ; \\
0 & \text { otherwise. }\end{cases}
$$

Proof. The Möbius function of $L_{O}(n, d)$ is

$$
\mu(P, Q)= \begin{cases}(-1)^{s} q^{\left(\begin{array}{c}
s \\
2
\end{array}\right)} & \text { if }\{0\} \neq P \leq Q \text { or } P=Q=\{0\} ; \\
\sum_{\{0\}<U \leq Q}-\mu(U, Q) & \text { if }\{0\}=P<Q ; \\
0 & \text { otherwise. }\end{cases}
$$

By Proposition 2.1, we have

$$
\sum_{\{0\}<U \leq Q}-\mu(U, Q)=\sum_{l=1}^{s}(-1)^{s-l+1}\left(\left[\begin{array}{l}
s \\
l
\end{array}\right]_{q}-\left[\begin{array}{c}
m \\
l
\end{array}\right]_{q}\right) q^{\left(\begin{array}{c}
s-l \\
2
\end{array}\right)} .
$$

Thus, the assertion follows.

Theorem 2.4. The characteristic polynomial of $L_{O}(n, d)$ is

$$
\begin{aligned}
& \chi\left(L_{O}(n, d), x\right)=x^{n}+\sum_{l=1}^{n}(-1)^{n-l+1}\left(\left[\begin{array}{l}
n \\
l
\end{array}\right]_{q}-\left[\begin{array}{l}
d \\
l
\end{array}\right]_{q}\right) q^{\left(\begin{array}{c}
n-l \\
2
\end{array}\right)} \\
& +\sum_{j=1}^{n-1} \sum_{t=\max \{0, d+j-n\}}^{\min \{d, j-1\}} \sum_{l=1}^{j}(-1)^{j-l+1} q^{(d-t)(j-t)+\left(\begin{array}{c}
j-l \\
2
\end{array}\right)}\left[\begin{array}{l}
d \\
t
\end{array}\right]_{q}\left[\begin{array}{c}
n-d \\
j-t
\end{array}\right]_{q}\left(\left[\begin{array}{l}
j \\
l
\end{array}\right]_{q}-\left[\begin{array}{l}
t \\
l
\end{array}\right]_{q}\right) x^{n-j} .
\end{aligned}
$$

Proof. By Proposition 2.1 and Lemma 2.3, we have

$$
\begin{aligned}
\chi\left(L_{O}(n, d), x\right)= & \sum_{P \in L_{O}(n, d)} \mu(\{0\}, P) x^{r_{O}\left(F_{q}^{n}\right)-r_{O}(P)} \\
= & x^{n}+\sum_{\{0\} \neq P \in L_{O}(n, d)} \mu(\{0\}, P) x^{n-\operatorname{dim}(P)} \\
= & x^{n}+\sum_{l=1}^{n}(-1)^{n-l+1}\left(\left[\begin{array}{l}
n \\
l
\end{array}\right]_{q}-\left[\begin{array}{l}
d \\
l
\end{array}\right]_{q}\right) q^{\left(\begin{array}{c}
n-l \\
2
\end{array}\right)} \\
& +\sum_{j=1}^{n-1} \sum_{t=\max \{0, d+j-n\}}^{\min \{d, j-1\}} \sum_{l=1}^{j}(-1)^{j-l+1} q^{(d-t)(j-t)+\left(\begin{array}{c}
j-l \\
2
\end{array}\right)}\left[\begin{array}{l}
d \\
t
\end{array}\right]_{q}\left[\begin{array}{c}
n-d \\
j-t
\end{array}\right]_{q}\left(\left[\begin{array}{l}
j \\
l
\end{array}\right]_{q}-\left[\begin{array}{l}
t \\
l
\end{array}\right]_{q}\right) x^{n-j} .
\end{aligned}
$$




\section{The Lattice $L_{R}(n, d)$}

In this section we prove that the lattice $L_{R}(n, d)$ is a finite atomic lattice, classify its geometricity and compute its characteristic polynomial.

Theorem 3.1. The following hold:

1) $L_{R}(n, d)$ is a finite atomic lattice.

2) $L_{R}(n, d)$ is geometric if and only if $n=2$.

Proof. 1) For any two elements $P, Q \in L_{R}(n, d), P \wedge Q=P+Q$ and

$$
P \vee Q= \begin{cases}P \cap Q & \text { if } P \cap Q \not \subset W ; \\ \{0\} & \text { otherwise. }\end{cases}
$$

Therefore $L_{R}(n, d)$ is a finite lattice. Note that $\{0\}$ is the unique minimum element. Let $P(n, d ; j)$ be the set of all the $j$-dimensional subspaces of $L_{R}(n, d)$, where $0 \leq j \leq n-1$. Then $P(n, d ; n-1)$ is the set of all the atoms in $L_{R}(n, d)$. In order to prove $L_{R}(n, d)$ is atomic, it suffices to show that every element of $P(n, d ; j)(0 \leq j \leq n-1)$ is a join of some atoms. The result is trivial for $j=n-1$. Suppose that the result is true for $j=n-l \leq n-1$. Let $U \in P(n, d ; n-l-1)$. By Proposition 2.1, the number of $n$-l-dimensional subspaces of $L_{R}(n, d)$ containing $U$ is equal to

$$
\left[\begin{array}{c}
l+1 \\
1
\end{array}\right]_{q}=\frac{q^{l+1}-1}{q-1} \geq 2 .
$$

Then there exist two different $(n-l)$-dimensional subspaces $U \subseteq U^{\prime}, U^{\prime \prime} \in L_{R}(n, d)$ such that $U=U^{\prime} \vee U^{\prime \prime}$. By induction $U$ is a join of some atoms. Therefore $L_{R}(n, d)$ is a finite atomic lattice.

2) For any $U \in L_{R}(n, d)$, we define $r_{R}(U)=n-\operatorname{dim} U$. It is routine to check that $r_{R}$ is the rank function on $L_{R}(n, d)$. It is obvious that $L_{R}(2,1)$ is a geometric lattice. Now assume that $n \geq 3$. Let $P$ be a 1 -dimensional subspace of $F_{q}^{n}$ and $P \subseteq W_{0}$. By Proposition 2.1, the number of 2-dimensional subspaces of $L_{R}(n, d)$ containing $P$ is equal to

$$
\left[\begin{array}{c}
n-1 \\
1
\end{array}\right]_{q}-\left[\begin{array}{c}
d-1 \\
1
\end{array}\right]_{q}=\frac{q^{d-1}\left(q^{n-d}-1\right)}{q-1} \geq 2 .
$$

Therefore, there exist two different 2-dimensional subspaces $P \subseteq P^{\prime}, P^{\prime \prime} \in L_{R}(n, d)$ such that $P=P^{\prime} \cap P^{\prime \prime}$. So $P^{\prime} \vee P^{\prime \prime}=\{0\}, P^{\prime} \wedge P^{\prime \prime}=P^{\prime}+P^{\prime \prime}$. Hence $r_{R}\left(P^{\prime} \vee P^{\prime \prime}\right)+r_{R}\left(P^{\prime} \wedge P^{\prime \prime}\right)=2 n-3>2 n-4=r_{R}\left(P^{\prime}\right)+r_{R}\left(P^{\prime \prime}\right)$, which implies that $L_{R}(n, d)$ is not a geometric lattice when $n \geq 3$.

Lemma 3.2. For any $P, Q \in L_{R}(n, d)$, suppose that $\operatorname{dim} P=t+s, \operatorname{dim} Q=t$ and $\operatorname{dim}\left(W_{0} \cap P\right)=m$. Then the Möbius function of $L_{R}(n, d)$ is

$$
\mu(P, Q)= \begin{cases}(-1)^{s} q^{\left(\begin{array}{c}
s \\
2
\end{array}\right)} & \text { if } P \leq Q \neq\{0\} \text { or } P=Q=\{0\} ; \\
\sum_{l=1}^{s}(-1)^{s-l+1}\left(\left[\begin{array}{l}
s \\
l
\end{array}\right]_{q}-\left[\begin{array}{c}
m \\
l
\end{array}\right]_{q}\right) q^{\left(\begin{array}{c}
s-l \\
2
\end{array}\right)} & \text { if } P<Q=\{0\} ; \\
0 & \text { otherwise. }\end{cases}
$$

Proof. The Möbius function of $L_{R}(n, d)$ is

$$
\mu(P, Q)= \begin{cases}(-1)^{s} q^{\left(\begin{array}{c}
s \\
2
\end{array}\right)} & \text { if } P \leq Q \neq\{0\} \text { or } P=Q=\{0\} ; \\
\sum_{P \leq U<\{0\}}-\mu(P, U) & \text { if } P<Q=\{0\} ; \\
0 & \text { otherwise. }\end{cases}
$$

Proposition 2.1 implies that 


$$
\sum_{P \leq U<\{0\}}-\mu(P, U)=\sum_{l=1}^{s}(-1)^{s-l+1}\left(\left[\begin{array}{l}
s \\
l
\end{array}\right]_{q}-\left[\begin{array}{c}
m \\
l
\end{array}\right]_{q}\right) q^{\left(\begin{array}{c}
s-l \\
2
\end{array}\right)} .
$$

Theorem 3.3. The characteristic polynomial of $L_{R}(n, d)$ is

$$
\chi\left(L_{R}(n, d), x\right)=x^{n}-1+\sum_{j=1}^{n}(-1)^{n-j}\left(\left[\begin{array}{l}
n \\
j
\end{array}\right]_{q}-\left[\begin{array}{l}
d \\
j
\end{array}\right]_{q}\right) q^{\left(\begin{array}{c}
n-j \\
2
\end{array}\right)}\left(x^{j}-1\right) .
$$

Proof. By Proposition 2.1, we have

$$
\begin{aligned}
\chi\left(L_{R}(n, d), x\right) & =\sum_{P \in L_{R}(n, d)} \mu\left(F_{q}^{n}, P\right) x^{r_{R}(\{0\})-r_{R}(P)} \\
& =x^{n}+\sum_{F_{q}^{n} \neq P \in L_{R}(n, d)} \mu\left(F_{q}^{n}, P\right) x^{\operatorname{dim}(P)} \\
& =x^{n}+\sum_{j=1}^{n}(-1)^{n-j}\left(\left[\begin{array}{c}
n \\
j
\end{array}\right]_{q}-\left[\begin{array}{c}
d \\
j
\end{array}\right]_{q}\right) q^{\left(\begin{array}{c}
n-j \\
2
\end{array}\right)} x^{j}+\sum_{l=1}^{n}(-1)^{n-l+1}\left(\left[\begin{array}{l}
n \\
l
\end{array}\right]_{q}-\left[\begin{array}{l}
d \\
l
\end{array}\right]_{q}\right) q^{\left(\begin{array}{c}
n-l \\
2
\end{array}\right)} \\
& =x^{n}-1+\sum_{j=1}^{n-1}(-1)^{n-j}\left(\left[\begin{array}{c}
n \\
j
\end{array}\right]_{q}-\left[\begin{array}{l}
d \\
j
\end{array}\right]_{q}\right) q^{\left(\begin{array}{c}
n-j \\
2
\end{array}\right)}\left(x^{j}-1\right) .
\end{aligned}
$$

\section{References}

[1] Aigner, M. (1979) Combinatorial Theory. Springer-Verlag, Berlin. http://dx.doi.org/10.1007/978-1-4615-6666-3

[2] Wan, Z. and Huo, Y. (2004) Lattices Generated by Transitive Sets of Subspaces Under Finite Classical Groups. 2nd Edition, Science Press, Beijing.

[3] Liu, Y.H. (2011) Special Lattice of Rough Algebras. Applied Mathematics, 2, 1522-1524. http://dx.doi.org/10.4236/am.2011.212215

[4] Huo, Y.J. and Wan, Z.-X. (2001) On the Geomericity of Lattices Generated by Orbits of Subspaces under Finite Classical Groups. Journal of Algebra, 243, 339-359. http://dx.doi.org/10.1006/jabr.2001.8819

[5] Gao, Y. and You, H. (2003) Lattices Generated by Orbits of Subspaces under Finite Singular Classical Groups and Its Characteristic Polynomials. Communications in Algebra, 31, 2927-2950. http://dx.doi.org/10.1081/AGB-120021900

[6] Wang, K.S. and Guo, J. (2009) Lattices Generated by Two Orbits of Subspaces Under Finite Classical Groups. Finite Fields and Their Applications, 15, 236-245. http://dx.doi.org/10.1016/j.ffa.2008.12.008

[7] Wang, K.S. and Li, Z.T. (2008) Lattices Associated with Vector Spaces Over a Finite Field. Linear Algebra and Its Applications, 429, 439-446. http://dx.doi.org/10.1016/j.laa.2008.02.035

[8] Guo, J., Li, Z.T. and Wang, K.S. (2009) Lattices Associated with Totally Isotropic Subspaces in Classical Spaces. Linear Algebra and Its Applications, 431, 1088-1095. http://dx.doi.org/10.1016/j.laa.2009.04.009

[9] Gao, S.G., Guo, J. and Liu, W. (2007) Lattices Generated by Strongly Closed Subgraphs in d-Bounded Distance-Regular Graphs. European Journal of Combinatorics, 28, 1800-1813. http://dx.doi.org/10.1016/j.ejc.2006.05.011

[10] Guo, J. and Gao, S.G. (2008) Lattices Generated by Join of Strongly Closed Subgraphs in d-Bounded Distance-Regular Graphs. Discrete Mathematics, 308, 1921-1929. http://dx.doi.org/10.1016/j.disc.2007.04.043

[11] Guo, J. and Wang, K.S. (2010) Posets Associated with Subspaces in a d-Bounded Distance-Regular Graph. Discrete Mathematics, 310, 714-719. http://dx.doi.org/10.1016/j.disc.2009.08.014

[12] Brouwer, A.E., Cohen, A.M. and Neumaier, A. (1989) Distance-Regular Graphs. Springer-Verlag, New York. http://dx.doi.org/10.1007/978-3-642-74341-2

[13] Wan, Z. (2002) Geometry of Classical Groups over Finite Fields. 2nd Edition, Science Press, Beijing/New York. 